\title{
Correlation of Isotope Count With Sentinel Node Positivity in Vulvar Cancer
}

\author{
Linn Woelber, MD, PhD, * Simon A. Joosse, PhD, † Donata Grimm, MD, * Sabrina Mathey, MD, * \\ Sven Mahner, MD, PhD, Eike Burandt, MD, PhD, $\S$ Susanne Klutmann, MD, PhD,// \\ Barbara Schmalfeldt, MD, PhD,* and Katharina Prieske, MD*
}

\begin{abstract}
Objective: Sentinel node biopsy (SNB) has become standard of care in early stage vulvar cancer. As the correlation of isotope count with the presence of metastases remains unclear, often several active nodes are excised per groin. This can result in increased morbidity in node-negative disease despite of SNB. In the current analysis, we assess whether resection of the hottest node could be sufficient to detect sentinel lymph node (SLN) metastasis.

Methods: Patients with primary vulvar cancer receiving an SNB with radioactive tracer at the University Medical Center Hamburg-Eppendorf between 2008 and 2015 were evaluated.

Results: A total of 145 patients with SNB were analyzed; thereof, 144 underwent bilateral SNB, resulting in 289 analyzed groins. A median of 2 SLNs (range, 1-7) per groin were removed. From 94 (32.5\%) of 289 groins, more than 2 SLNs were excised. Median overall SLN isotope count was $1400 \mathrm{cps}$. In 50 groins, a positive SLN was detected (unilateral in 38 patients, bilateral in 6). The median number of positive SLN per groin was 1 (range, 1-4). The SLN with the highest isotope count carried metastases in 36 (78.3\%) of 46 groins (in 4 cases, the highest count was unknown). In 10 (21.7\%) of 46 positive groins, the SLN with the highest count was not the metastatic SLN (9/10 second highest count). Median count of these 10 SLN was $60 \%$ of the highest count with a range from $11.0 \%$ to $74.0 \%$.

Conclusions: The highest isotope count does not reliably detect the positive SLN in vulvar cancer. To prevent mostly fatal groin recurrences, surgeons should continue to remove all SLN accumulating relevant radioactive tracer over background activity.
\end{abstract}

Key Words: Vulvar cancer, Sentinel, Groin, Isotope count, Metastases

Received December 28, 2017, and in revised form February 20, 2018.

Accepted for publication March 23, 2018.

(Int J Gynecol Cancer 2018;28: 1403-1409)

$S$ ince publication of the results of the Groningen International $\checkmark$ Study on Sentinel Nodes in Vulvar Cancer (GROINSS-V1) in 2008 and the Gynecological Oncology Group 173 trial in
2012, sentinel node biopsy (SNB) is a widely accepted and reliable surgical staging method for clinically node-negative early stage vulvar cancer. ${ }^{1,2}$ In general, the sentinel lymph
Departments of *Gynecology and Gynecologic Oncology, and $\uparrow$ Tumor Biology, University Medical Center Hamburg-Eppendorf; tDepartment of Obstetrics and Gynecology, Ludwig-MaximilliansUniversity Munich, Munich; §Institute of Pathology; and ||Department for Diagnostic and Interventional Radiology and Nuclear Medicine, University Medical Center Hamburg-Eppendorf, Hamburg, Germany.

Copyright (C) 2018 by IGCS and ESGO

ISSN: $1048-891 \mathrm{X}$

DOI: $10.1097 /$ IGC.0000000000001298
Address correspondence and reprint requests to Linn Woelber, MD, $\mathrm{PhD}$, Department of Gynecology and Gynecologic Oncology, University Medical Center Hamburg-Eppendorf, Martinistraße 52, 20246 Hamburg, Germany. E-mail: lwoelber@uke.de.

This study was funded by internal departmental sources.

The authors declare no conflicts of interest.

Supplemental digital content is available for this article. Direct URL citation appears in the printed text and is provided in the HTML and PDF versions of this article on the journal's Web site (www.ijgc.net). 
node (SLN) is defined as the first node draining lymphatic fluid from the tumor site. Consequently, if nodal metastases occur, the tumor cells travel to the SLN and can ideally be diagnosed via biopsy of this previously marked node. ${ }^{3}$ Sentinel lymph node identification in patients with vulvar cancer has been first described by Levenback et al., ${ }^{4,5}$ In the early 1990 s, they demonstrated the feasibility of intraoperative lymphatic mapping by administration of isosulfan blue dye in 2 case series of 9 and subsequently 21 patients. ${ }^{4,5}$ However, larger studies from other tumor entities such as cutaneous melanoma and breast cancer showed that the combination of intraoperatively administered blue dye and preoperatively injected radioactive tracer $(\mathrm{Tc} 99 \mathrm{~m})$ with intraoperative gamma probe detection is superior to blue dye alone. ${ }^{6-8}$ With the combined technique, the SLN identification rate is generally very high with a sensitivity of $95 \%$ (95\% confidence interval [CI], 92\%-98\%) and a negative predictive value of 97.9.9.10 Ideally, with the SLN technique, only 1 node should be identified for excision to keep morbidity low. In vulvar cancer however, often multiple, up to 7 SLN nodes per groin are identified ${ }^{11}$ and are consequently excised to guide further groin treatment. ${ }^{12,13}$ Thereby, morbidity potentially is increased with recurrent seroma, lymphedema, wound breakdown, or erysipelas despite of node-negative disease. Theoretically, the SLN with the highest isotope count ("hottest" SLN) should be most likely to harbor tumor cells. However, in breast cancer, the false-negative rate for detection of SLN metastases was shown to be very variable if only the hottest SLN is removed (range, 9\%-29\%). ${ }^{14-20}$ Currently, the $10 \%$ rule is the most widely accepted in breast cancer, meaning that all nodes with more than $10 \%$ of the ex vivo isotope count of hottest SLN should be removed, lowering the false-negative rate to $2.5 \%$ to $6.4 \% .{ }^{14,19,20}$ The $10 \%$ rule was also applied in 1184 early stage cutaneous melanoma patients subjected to SNB. ${ }^{21}$ The false-negative rate significantly decreased from $13.9 \%$ to $0.4 \%$ by using the $10 \%$ rule compared with removing the hottest SLN only.

In vulvar cancer, the correlation of the isotope count with the presence of metastases remains unclear. The aim of this analysis was therefore to assess whether resection of the hottest node would be sufficient to reliably detect SLN metastases in vulvar cancer.

\section{MATERIALS AND METHODS}

\section{Patient Population}

Consecutive patients with squamous cell vulvar cancer undergoing successful SNB with radioactive tracer from 2008 to 2015 at the Gynecological Cancer Center in Hamburg, Germany were included in the analysis. Informed consent had been obtained from all included patients to review their medical records according to our investigational review board and ethics committee guidelines (Ethics Committee of the Medical Board Hamburg reference number 190504). The institutional approach to the surgical treatment of vulvar cancer during the investigational period consisted of radical excision of the primary tumor; the SLN procedure was offered to patients with locally restricted disease and clinically negative groin nodes according to the inclusion criteria of the
GROINSS study published in 2008. ${ }^{1}$ Three patients originally planned for SNB were subsequently excluded from the analysis. In the first patient, bilateral SNB was not successful owing to prior surgery in 1 groin. Instead she received an inguinofemoral lymphadenectomy (LAE) in that groin. In the other 2 patients, no SLN could be identified in planar lymphoscintigraphy preoperatively. Intraoperatively, lymph nodes were suspicious and metastatic in frozen section so that bilateral inguinofemoral LAE was performed. All pathological studies were performed by specialized gynecopathologists. Clinical outcome was followed from the date of primary surgery to the date of death or until June 2016. For tumor staging, the International Federation of Gynecology and Obstetrics stage groupings as well as the International Union Against Cancer tumor-node-metastasis classification sixth edition were used for homogeneity. ${ }^{22,23}$

\section{Sentinel Node Detection}

The day before surgery, all patients received a conventional planar lymphoscintigraphy of the groins. For that purpose, 4 peritumoral intradermal deposits $(0.2-0.3 \mathrm{~mL}$ volume each) at 3,6,9, and 12 o'clock were placed, using a 27 -gauge needle. An overall mean $\pm \mathrm{SD}$ dosage of $85 \pm 12 \mathrm{MBq}$ $99 \mathrm{mTc}$-nanocolloid was injected. One hour after injection, anterior and lateral static views were obtained for planar lymphoscintigraphy. The sites of the SLNs were marked on the skin. Images were made using a large field-of-view dual-head gamma scintillation camera (ECAM, Siemens Medical Solutions, Hoffman Estates, Ill) equipped with a low-energy high-resolution parallel-hole collimator with the following imaging parameters: matrix size of $256 \times 256$ and energy window at $141 \mathrm{keV}$ with $15 \%$ width, 5 minutes each.

On the following day, a handheld gamma counter (Navigator GPS, RMD Instruments) was used intraoperatively to identify the SLNs. After removal of the first SLN, the groin was reexamined for residual radioactivity, and potentially further SLNs were removed. Radioactivity was measured, and the respective activity was documented for every single node in the study. Identified SLNs were sent for frozen section to the gynecopathologists; if they came back negative, no further node dissection was performed in the majority of patients. Ultrastaging of all negative SLNs obtained was performed postoperatively according to the GROINSS-VI study to identify further metastases as described previously. ${ }^{1}$ In case of positive SLN in ultrastaging, a systematic unilateral or bilateral LAE was performed during a second surgical intervention.

\section{Statistical Analysis}

Difference in median isotype count was calculated by Wilcoxon rank sum test. Survival probabilities were calculated by Kaplan-Meier curves and log-rank test. An $\alpha$ level of 0.05 was used to assess statistical significance.

\section{RESULTS}

\section{Patient Characteristics}

A total of 145 patients with successful SNB (144 bilateral and 1 unilateral SNB) during the investigational period 
TABLE 1. Patient characteristics

\begin{tabular}{|c|c|c|c|c|c|c|}
\hline Characteristics & Total $(n=145)$ & Total (\%) & No $(n=100)$ & No \% & $N+(n=45)$ & $\mathrm{N}+(\%)$ \\
\hline $\begin{array}{l}\text { Age at FD, median, y } \\
\text { (range) }\end{array}$ & $\begin{array}{c}57 \\
(20-88)\end{array}$ & n.a. & $\begin{array}{c}57 \\
(20-85)\end{array}$ & n.a. & $\begin{array}{c}56 \\
(26-88)\end{array}$ & n.a. \\
\hline \multicolumn{7}{|l|}{ Tumor stage } \\
\hline pT1b & 116 & 80 & 84 & 84 & 33 & 73.3 \\
\hline pT1a & 5 & 2.7 & 4 & 4 & 0 & 0 \\
\hline pT2 & 20 & 13.7 & 11 & 11 & 9 & 19.6 \\
\hline pT3 & 4 & 2.7 & 1 & 1 & 3 & 6.5 \\
\hline Unilateral groin metastases & 39 & 26.9 & n.a. & n.a. & 39 & 86.7 \\
\hline Bilateral groin metastases & 6 & 4.14 & n.a. & n.a. & 6 & 13.3 \\
\hline Unilateral SLN metastases & 38 & 26.2 & n.a. & n.a. & 38 & 84.4 \\
\hline Bilateral SLN metastases & 6 & 4.14 & n.a. & n.a. & 6 & 13.3 \\
\hline Median tumor diameter, $\mathrm{mm}$ & 18 & n.a. & 14 & n.a. & 21 & n.a. \\
\hline (range) & $(1.2-75)$ & n.a. & $(1.2-65)$ & n.a. & $(2-75)$ & n.a. \\
\hline Unknown & 10 & n.a. & 9 & n.a. & 1 & n.a. \\
\hline Median depth of invasion, $\mathrm{mm}$ & 3 & n.a. & 2.6 & n.a. & 4 & n.a. \\
\hline (range) & $(0.4-27)$ & n.a. & $(0.4-18)$ & n.a. & $(1.1-27)$ & n.a. \\
\hline Unknown & 9 & n.a & 6 & n.a. & 3 & n.a \\
\hline Median size of lymph node metastasis, mm & 2.5 & n.a. & n.a. & n.a. & 2.5 & n.a. \\
\hline (range) & $0.15-26$ & n.a. & n.a. & n.a. & $0.15-26$ & n.a. \\
\hline Unknown & 6 & n.a & n.a & n.a & 6 & n.a \\
\hline \multicolumn{7}{|l|}{ Resection status of vulvar primary } \\
\hline R0 & 140 & 96.6 & 97 & 97 & 43 & 95.6 \\
\hline $\mathrm{R} 1$ & 0 & 0 & 0 & 0 & 0 & 0 \\
\hline VIN in margin & 4 & 2.7 & 2 & 2 & 2 & 4.35 \\
\hline Unknown & 1 & 0.68 & 1 & 1 & 0 & 0 \\
\hline \multicolumn{7}{|l|}{ Grading } \\
\hline G1 & 6 & 4.1 & 6 & 6 & 0 & 0 \\
\hline G2 & 99 & 68.3 & 71 & 71 & 28 & 62.2 \\
\hline G3 & 38 & 26 & 21 & 21 & 17 & 37 \\
\hline Unknown & 2 & 1.4 & 2 & 2 & 0 & 0 \\
\hline \multicolumn{7}{|l|}{ Vulvar surgery } \\
\hline Vulvectomy & 11 & 7.5 & 5 & 5 & 6 & 13 \\
\hline Radical local excision & 133 & 91.7 & 94 & 94 & 39 & 86.7 \\
\hline No local surgery & 1 & 0.68 & 1 & 1 & 0 & 0 \\
\hline Pelvic node dissection & 1 & 0.68 & 0 & 0 & 1 & 2.2 \\
\hline \multicolumn{7}{|l|}{ Adjuvant therapy } \\
\hline Radiotherapy & 15 & 11 & 0 & 0 & 15 & 34.8 \\
\hline Chemoradiation & 4 & 2.8 & 0 & 0 & 4 & 8.9 \\
\hline Laser & 2 & 1.37 & 2 & 2 & 0 & 0 \\
\hline \multicolumn{7}{|l|}{ Radiation field } \\
\hline Vulva/ \pm groin $/ \pm$ pelvis & 17 & 11.7 & 1 & 1 & 17 & 37.8 \\
\hline Groin only & 3 & 2.1 & 0 & 0 & 3 & 6.7 \\
\hline No adjuvant therapy & 124 & 85 & 99 & 99 & 25 & 54.4 \\
\hline
\end{tabular}


TABLE 1. (Continued)

\begin{tabular}{lcccccc}
\hline Characteristics & Total $(\mathbf{n}=\mathbf{1 4 5})$ & Total $(\mathbf{\%})$ & $\mathbf{N 0}(\mathbf{n}=\mathbf{1 0 0})$ & $\mathbf{N 0} \%$ & $\mathbf{N}+(\mathbf{n}=\mathbf{4 5})$ & $\mathbf{N}+(\mathbf{\%})$ \\
\hline Neoadjuvant RTX & 1 & 0.68 & 1 & 1 & 0 & 0 \\
Median FU, mo & 30 & n.a. & 31.5 & n.a. & 28.5 & n.a. \\
$\quad($ range $)$ & $(0.1-101)$ & n.a. & $(0.1-101)$ & n.a. & $(1-73)$ & n.a. \\
\hline
\end{tabular}

n.a., not applicable; FD, first diagnosis; RTX, chemoradiation.

(2008-2015) were included in this analysis, resulting in 289 analyzed groins. Median follow-up (FU) was 30 months (range, 0.1-101 months). Detailed patient characteristics are listed in Table 1. Four patients received bilateral SNB despite a pT1a vulvar cancer due to lymphangioinvasion diagnosed in the primary tumor; the excised SLNs of these were all negative. Ten patients presented with a primary tumor size greater than $40 \mathrm{~mm}$ (range, 41-75 mm; median, $55 \mathrm{~mm}$ ) in definitive histology; 4 of these had a positive SLN and received a consecutive inguinofemoral LAE (3 bilateral, 1 unilateral). After a median FU of 21 months, none of those 10 patients had developed a groin recurrence. However, 1 patient suffered from distant metastases to the lungs and died 19 months later.

\section{Groin Characteristics}

The majority of patients in this analysis received an SNB only $(\mathrm{n}=101,69.2 \%)$. Of these, 100 were node negative and did not require further treatment (Table 2). Median number of excised SLNs per groin was 2 (287 groins; range, $1-7$; in 2 groins, the number of excised SLN was unknown) (Table 2). However, in $94(32.5 \%)$ of 289 groins, more than 2 SLNs were excised. In $50(17.3 \%)$ of 289 groins, a positive SLN was detected (unilateral in 38 patients, bilateral in 6). The median number of positive SLN per groin was 1 (range, $1-4)$. A total of 45 patients presented with lymph node metastases (39 patients unilateral, 6 bilateral); thereof, 1 had a non-SLN metastasis only. Of 44 SLN-positive patients, 36 patients had a second bilateral and 7 patients had a unilateral inguinofemoral LAE owing to small strictly unilateral tumors or ipsilateral micrometastasis with contralateral negative SLN. None of these 7 patients presented with a groin recurrence after a median of 21 months. One patient presented with a $1.5-\mathrm{mm}$ SLN metastasis but did not receive a consecutive inguinofemoral LAE owing to comorbidities. This patient presented in a poor general condition and died owing to a stroke 11 months after diagnosis. In patients undergoing a complete groin dissection, a median of 10 lymph nodes per groin were dissected (range, 1-23; 83 groins). The median number of positive lymph nodes per groin was 1 in node positive patients (range, $1-5 ; 51$ groins).

Three patients suffered from isolated groin recurrence after 5, 17, and 25 months. Thereof, 1 patient initially presented with microinvasive disease (pT1a) and negative bilateral SLN. She suffered from groin recurrence 25 months after initial diagnosis and received only unilateral complete groin dissection and radiation owing to comorbidities. She died from vulvar cancer 5 months after groin recurrence. The second patient was 66 at first diagnosis and presented with a pT1b squamous cell tumor (14 mm, G3). She received a bilateral SNB (0/4SLN) and wide excision of the tumor. Unilateral groin recurrence occurred after 5 months. The third patient was 48 at initial diagnosis and also presented with a pT $1 \mathrm{~b}, 10 \mathrm{~mm}$ squamous cell vulvar cancer. She received a wide excision and SNB (0/3SLN). Groin recurrence occurred 17 months after first diagnosis. Both patients received bilateral radical groin dissection and consecutive chemoradiation with cisplatin $\left(40 \mathrm{mg} / \mathrm{m}^{2}\right)$ to the groins and pelvis. They were still alive with no evidence of disease at 22 and 49 months after groin recurrence, respectively. Both patients received bilateral complete groin dissection and consecutive chemoradiation to the groins with cisplatin. They were still alive at last FU 22 and 49 months after groin recurrence, respectively.

Two- and 3-year disease-free survival was $86.1 \%(95 \%$ CI, 0.79-0.94) and $80.7 \%(95 \%$ CI, 0.72-0.9) in nodenegative patients with SNB only $(\mathrm{n}=100)$ and $77.5 \%$ (95\% CI, 0.65-0.92) and 74.4\% (95\% CI, 0.61-0.89) in nodepositive patients $(\mathrm{n}=45)$, respectively $(P=0.33$ for 3-year disease-free survival; Fig. 1). Five-year OS was $90.9 \%$ (95\% CI, 0.84-0.98) in node-negative and $82.6 \%$ (95\% CI, $0.69-0.99)$ in node-positive patients.

\section{Sentinel Isotope Count}

Median overall SLN isotope count was $1400 \mathrm{cps}$. There was no difference of median count in negative compared with positive SLN (median count 1396 cps vs $1614 \mathrm{cps} ; P=0.90$ ). The SLN with the highest isotope count carried metastases in $36(78.0 \%)$ of 46 groins (in 4 cases the highest count was unknown). In 10 (25.0\%) of 46 positive groins, the SLN with the highest count was not the metastatic SLN (in 9/10 second highest count). Median count of these 10 SLN was $60 \%$ of the highest count with a range from $11.0 \%$ to $74.0 \%$ (Supplementary Table 1, http://links.lww.com/IGC/A752).

\section{DISCUSSION}

Despite numerous studies that confirmed the accuracy of SNB for assessment of the nodal status, the optimum technique of SNB in vulvar cancer still remains a matter of debate. In vulvar cancer, high background activity often leads to removal of greater than 2 SLNs and therefore might increase morbidity despite node-negative disease. The present analysis shows that the highest isotope count correlates with SLN metastases in the majority of cases (75\%); however, it does not reliably predict SLN metastases in all patients. With the application of the $10 \%$ rule suggested by other authors primarily for breast cancer or malignant melanoma, ${ }^{14,19-21}$ no SLN metastases would have been missed in our cohort (lowest 
TABLE 2. Groin characteristics

\begin{tabular}{|c|c|c|}
\hline Groin Characteristics & $N=145$ Patients & $\%$ \\
\hline \multicolumn{3}{|l|}{ Groin surgery } \\
\hline \multicolumn{3}{|l|}{ Full groin dissection (unilateral or bilateral) } \\
\hline $\mathrm{SLN}^{+}$followed by unilateral groin dissection & 7 & 4.8 \\
\hline $\mathrm{SLN}^{+}$followed by bilateral groin dissection & 36 & 24.8 \\
\hline $\mathrm{SLN}^{+}$no groin dissection due to comorbidity & 1 & 0.69 \\
\hline Non-SLN positive followed by bilateral groin dissection & 1 & 0.69 \\
\hline \multicolumn{3}{|l|}{ SLN } \\
\hline Patients with unilateral excised SLN & 1 & 0.69 \\
\hline Patients with bilateral excised SLN & 144 & 99.3 \\
\hline Patients with unilateral positive SLN & 38 & 26.2 \\
\hline Patients with bilateral positive SLN & 6 & 4.1 \\
\hline \multicolumn{3}{|l|}{ SLN counts per groin ( 46 groins plus 4 unknown count) } \\
\hline Groins with positive SLN highest count & 36 & 78.3 \\
\hline Groins with other positive SLN & 10 & 21.7 \\
\hline Groins with SLN count unknown & 4 (4/50 groins) & 8 \\
\hline \multicolumn{3}{|l|}{ SLN counts } \\
\hline Counts $\mathrm{SLN}^{+}$(median) range & $1614(74-10,721)$ & n.a. \\
\hline Counts $\mathrm{SLN}^{-}$(median) range & $1396(4-17,412)$ & n.a. \\
\hline \multicolumn{3}{|c|}{ Excised lymph nodes per groin (only pat. with full groin dissection) } \\
\hline Median (range) & $10(1-23)$ & n.a. \\
\hline No. groins & 81 & n.a. \\
\hline \multicolumn{3}{|c|}{ Positive lymph nodes per groin (only pat. with full groin dissection) } \\
\hline Median (range) & $1(1-5)$ & n.a. \\
\hline No. groins & 51 & n.a. \\
\hline \multicolumn{3}{|l|}{ Excised sentinel nodes/groin } \\
\hline Median (range) & $2(1-7)$ & n.a. \\
\hline No. groins & 287 & n.a. \\
\hline No. excised sentinel nodes unknown & 2 & n.a. \\
\hline \multicolumn{3}{|l|}{ Positive sentinel nodes/groin } \\
\hline Median (range) & $1(1-4)$ & n.a. \\
\hline No. groins & 50 & n.a. \\
\hline Isolated groin recurrence & 3 & 2.1 \\
\hline
\end{tabular}

isotope count of the SLN that contained metastases: $11 \%$ of the highest count). In our cohort, only 1 patient presented with an SLN metastases in the third hottest SLN node, which still showed $25 \%$ of the isotope count of the hottest SLN (269/1073 cps). There is no clear consensus in national guidelines regarding the number of SLN nodes that should be excised, and intraoperatively surgeons are often faced with the decision to remove a fourth or fifth mildly active node. However, the $10 \%$ rule was already part of the sentinel node detection protocol in the GROINSS-VI studies. After removal of the sentinel nodes, the biopsy bed was reexamined for radioactivity, and if higher than $10 \%$ of the first excised lymph node, the dissection was continued in search of additional sentinel nodes. Protocols for the absolute dose of 99Tc nanocolloid injection have largely been adapted from breast cancer and melanoma. They differ between countries and institutions (50-100 MBq total dose per injection spot). In vulvar cancer, the SLN procedure has initially been performed combining radioactive tracer and blue dye and therewith yielded highly accurate detection rates $(98 \%-100 \%){ }^{24} \mathrm{New}$ guidelines do not mandatory require blue dye. ${ }^{25,26}$ Compared with breast cancer and melanoma, the SLN detection rate in vulvar cancer is even more accurate. In a meta-analysis of 8059 patients with breast cancer, $96 \%$ had successfully mapped SLNs. However, in more than $50 \%$ of included breast cancer studies, detection rates of greater than $90 \%$ were reported. ${ }^{27}$ A possible explanation for the higher identification rate in vulvar cancer ${ }^{24}$ might be the anatomy of the lymphatic channels to the inguinofemoral 


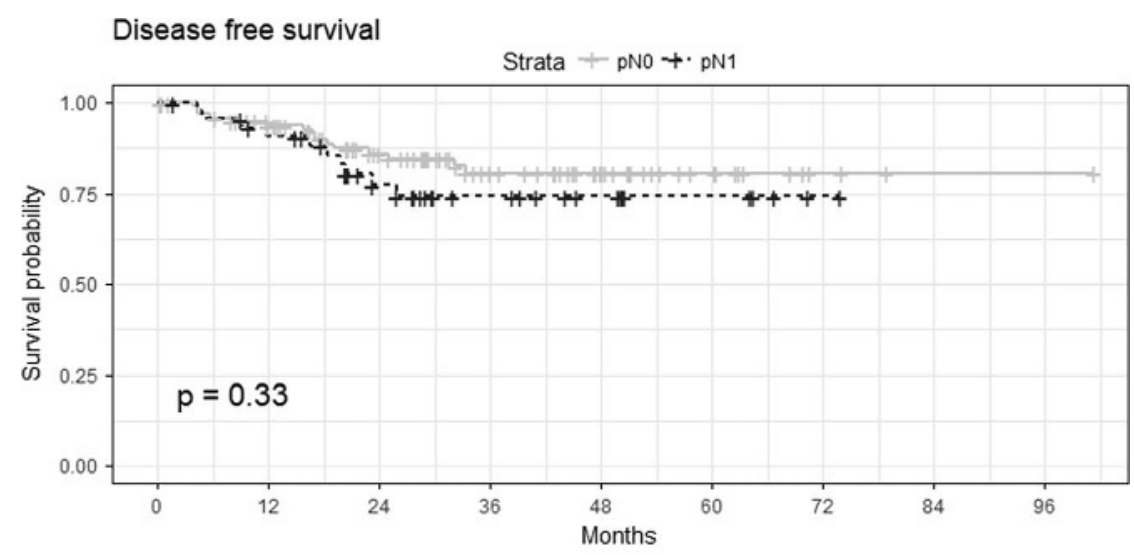

\begin{tabular}{|c|c|c|c|c|c|c|c|c|c|}
\hline \multirow{4}{*}{ 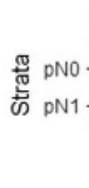 } & \multicolumn{9}{|c|}{ Number at risk } \\
\hline & 100 & 85 & 59 & 38 & 24 & 11 & 3 & 1 & 1 \\
\hline & 45 & 38 & 25 & 15 & 10 & 5 & 1 & 0 & 0 \\
\hline & 0 & 12 & 24 & 36 & $\begin{array}{l}48 \\
\text { Mon }\end{array}$ & 60 & 72 & 84 & 96 \\
\hline
\end{tabular}

FIGURE 1. Kaplan-Meyer curve of disease free survival of $\mathrm{pN} 0$ versus $\mathrm{pN} 1$ patients.

nodes, which seem to vary only minimally, and the SLN is almost always located above the cribriform fascia of the fossa ovalis. The nodal recurrence risk, however, seems less favorable in vulvar cancer when compared with breast cancer $(2.3 \%$ vs $0.1 \%-0.3 \%){ }^{28}$ This might be partly explained by the fact that the majority of breast cancer patients will receive adjuvant treatment, whereas node-negative vulvar cancer patients will not. Therefore, the accuracy of SLN detection technique is even more important, as lethality of nodal recurrence in vulvar cancer is high. Some authors proposed the use of bigger particles like colloidal albumin or (99m)Tcphytate in comparison with Technetium. Sentinel lymph node number is closely related to the size of the radiocolloid used, ${ }^{29}$ and bigger particles might not get so easily into other nodes than into the sentinel node. New surgical techniques to identify the SLN may be of additional value for intraoperative imaging of the SLN in vulvar cancer. Recently, intraoperative near-infrared fluorescence (NIRF) imaging was introduced as a new technique for SLN detection. This approach is based on the intraoperative injection of a fluorescent agent around the primary tumor, which will flow through the lymphatic vessels and accumulate in the SLN(s). Upon excitation with a laser beam, the agent emits light of a longer wavelength, which is captured and processed by a fluorescence camera. Real-time images can be displayed on monitors in the operating theatre. Currently, the fluorescent agent of choice is indocyanin green, a Food and Drug Administration-approved agent with little toxicity that has been used for decades for ophthalmic angiography and evaluation of liver perfusion. In different pilot studies, this technique has been investigated for gynecological malignancies, for example, vulvar cancer. ${ }^{30-34}$ In 70 patients with vulvar cancer that have been described in 5 clinical studies, $96.6 \%$ of SLN detected by $99 \mathrm{mTc}$ nanocolloid were also fluorescent in vivo. This was in contrast to blue dye, which stained only $70.6 \%$ of the SLNs $(P<0.001)$. However, in a pilot study by Crane et al, ${ }^{30}$ detection rates in obese patients were limited, owing to the limited penetration depth of NIRF. In total, 29 SLNs were detected by radiocolloid, of which 26 were also detected by fluorescence and 21 were blue. The authors conclude that these first clinical results indicate that intraoperative transcutaneous lymphatic mapping using fluorescence is technically feasible in a subgroup of lean vulvar cancer patients. Currently, indocyanin green is the only Food and Drug Administration-approved NIRF agent. Future research on intraoperative fluorescence imaging for SLN detection may benefit from fluorescent agents with stronger penetration properties. Results of larger clinical studies especially concerning the oncologic safety (false-negative rate) and number of removed SLNs are still pending.

This retrospective analysis shows that the hottest SLN has the highest likelihood of harboring the tumor cells in vulvar cancer. In all other cases except for one, the second hottest SLN contained the tumor cells. However, because groin recurrences in vulvar cancer are almost always fatal, oncologic safety has to be balanced carefully against the potentially increased morbidity of a fourth or fifth excised sentinel. Based on the results of this study, it is not sufficient to remove the hottest sentinel node only and surgeons should follow the $10 \%$ rule known from other entities as a guidance.

\section{REFERENCES}

1. Van der Zee AG, Oonk MH, De Hullu JA, et al. Sentinel node dissection is safe in the treatment of early-stage vulvar cancer. $J$ Clin Oncol. 2008;26:884-889.

2. Levenback CF, Ali S, Coleman RL, et al. Lymphatic mapping and sentinel lymph node biopsy in women with squamous cell carcinoma of the vulva: a gynecologic oncology group study. $J$ Clin Oncol. 2012;30:3786-3791.

3. Morton DL, Wen DR, Wong JH, et al. Technical details of intraoperative lymphatic mapping for early stage melanoma. Arch Surg. 1992;127:392-399. 
4. Levenback C, Burke TW, Gershenson DM, et al. Intraoperative lymphatic mapping for vulvar cancer. Obstet Gynecol. 1994;84:163-167.

5. Levenback C, Burke TW, Morris M, et al. Potential applications of intraoperative lymphatic mapping in vulvar cancer. Gynecol Oncol. 1995;59:216-220.

6. Albertini JJ, Lyman GH, Cox C, et al. Lymphatic mapping and sentinel node biopsy in the patient with breast cancer. Jama. 1996;276:1818-1822.

7. Krag DN, Meijer SJ, Weaver DL, et al. Minimal-access surgery for staging of malignant melanoma. Arch Surg. 1995;130:654-658; discussion 9-60.

8. Kapteijn BA, Nieweg OE, Liem I, et al. Localizing the sentinel node in cutaneous melanoma: gamma probe detection versus blue dye. Ann Surg Oncol. 1997;4:156-160.

9. de Hullu JA, Doting E, Piers DA, et al. Sentinel lymph node identification with technetium-99m-labeled nanocolloid in squamous cell cancer of the vulva. J Nucl Med. 1998;39:1381-1385.

10. Meads C, Sutton AJ, Rosenthal AN, et al. Sentinel lymph node biopsy in vulval cancer: systematic review and meta-analysis. Br J Cancer. 2014;110:2837-2846.

11. Woelber L, Eulenburg C, Grimm D, et al. The risk of contralateral non-sentinel metastasis in patients with primary vulvar cancer and unilaterally positive sentinel node. Ann Surg Oncol. 2016;23:2508-2514.

12. Mahner S, Jueckstock J, Hilpert F, et al. Adjuvant therapy in lymph node-positive vulvar cancer: the AGO-CaRE-1 study. J Natl Cancer Inst. 2015;107. doi: 10.1093/jnci/dju426.

13. Klapdor R, Hillemanns $P$, Wolber L, et al. Outcome after sentinel lymph node dissection in vulvar cancer: a subgroup analysis of the AGO-CaRE-1 study. Ann Surg Oncol. 2017;24:1314-1321.

14. Bourgeois P, Nogaret JM, Veys I, et al. How 'hot' is the pathologically positive sentinel lymph node in breast cancer patients? Nucl Med Commun. 2003;24:513-518.

15. Wong SL, Edwards MJ, Chao C, et al. Sentinel lymph node biopsy for breast cancer: impact of the number of sentinel nodes removed on the false-negative rate. J Am Coll Surg. 2001;192:684-689; discussion 9-91.

16. Tafra L, Lannin DR, Swanson MS, et al. Multicenter trial of sentinel node biopsy for breast cancer using both technetium sulfur colloid and isosulfan blue dye. Ann Surg. 2001;233:51-59.

17. Borgstein PJ, Pijpers R, Comans EF, et al. Sentinel lymph node biopsy in breast cancer: guidelines and pitfalls of lymphoscintigraphy and gamma probe detection. $\mathrm{J} \mathrm{Am} \mathrm{Coll}$ Surg. 1998;186:275-283.

18. Rink T, Heuser T, Fitz H, et al. Lymphoscintigraphic sentinel node imaging and gamma probe detection in breast cancer with Tc-99m nanocolloidal albumin: results of an optimized protocol. Clin Nucl Med. 2001;26:293-298.

19. Martin RC 2nd, Edwards MJ, Wong SL, et al. Practical guidelines for optimal gamma probe detection of sentinel lymph nodes in breast cancer: results of a multi-institutional study. For the University of Louisville Breast Cancer Study Group. Surgery. 2000;128:139-144.
20. Camp ER, Cendan JC, Feezor R, et al. The hottest sentinel lymph node is not always the positive node. Am Surg. 2004;70:475-478; discussion 8.

21. McMasters KM, Reintgen DS, Ross MI, et al. Sentinel lymph node biopsy for melanoma: how many radioactive nodes should be removed? Ann Surg Oncol. 2001;8:192-197.

22. Beller U, Quinn MA, Benedet JL, et al. Carcinoma of the vulva. FIGO 26th Annual Report on the Results of Treatment in Gynecological Cancer. Int J Gynaecol Obstet. 2006;95(suppl 1): S7-S27.

23. Benedet JL, Bender H, Jones H 3rd, et al. FIGO staging classifications and clinical practice guidelines in the management of gynecologic cancers. FIGO Committee on Gynecologic Oncology. Int J Gynaecol Obstet. 2000;70:209-262.

24. de Hullu JA, Hollema H, Piers DA, et al. Sentinel lymph node procedure is highly accurate in squamous cell carcinoma of the vulva. J Clin Oncol. 2000;18:2811-2816.

25. Schnürch HG, Ackermann S, Alt CD, et al. Diagnosis, therapy and follow-up care of vulvar cancer and its precursors. Guideline of the DGGG and DKG (S2k-Level, AWMF Registry Number 015/059, November 2015. Geburtshilfe Frauenheilkd. 2016;76:1035-1046.

26. ESGO Guidelines Vulvar Cancer. 2016. Available at: https:// guidelinesesgoorg/media/2016/08/ESGO-Vulvar-cancerComplete-report-fxd2pdf. Accessed September 13, 2016.

27. Kim T, Giuliano AE, Lyman GH. Lymphatic mapping and sentinel lymph node biopsy in early-stage breast carcinoma: a metaanalysis. Cancer. 2006;106:4-16.

28. de Hullu JA, Oonk MH, Ansink AC, et al. Pitfalls in the sentinel lymph node procedure in vulvar cancer. Gynecol Oncol. 2004;94:10-15.

29. Wilhelm AJ, Mijnhout GS, Franssen EJ. Radiopharmaceuticals in sentinel lymph-node detection - an overview. Eur J Nucl Med. 1999;26:S36-S42.

30. Crane LM, Themelis G, Arts HJ, et al. Intraoperative near-infrared fluorescence imaging for sentinel lymph node detection in vulvar cancer: first clinical results. Gynecol Oncol. 2011;120:291-295.

31. Schaafsma BE, Verbeek FP, Peters AA, et al. Near-infrared fluorescence sentinel lymph node biopsy in vulvar cancer: a randomised comparison of lymphatic tracers. BJOG. 2013;120:758-764.

32. Matheron HM, van den Berg NS, Brouwer OR, et al. Multimodal surgical guidance towards the sentinel node in vulvar cancer. Gynecol Oncol. 2013;131:720-725.

33. Hutteman M, van der Vorst JR, Gaarenstroom KN, et al. Optimization of near-infrared fluorescent sentinel lymph node mapping for vulvar cancer. Am J Obstet Gynecol. 2012;206:89 e1-5.

34. Handgraaf HJ, Verbeek FP, Tummers QR, et al. Real-time near-infrared fluorescence guided surgery in gynecologic oncology: a review of the current state of the art. Gynecol Oncol. 2014;135:606-613. 